\title{
Biologische Einflussfaktoren auf die Knochenbruchheilung
}

Melanie Haffner-Luntzer, Anita Ignatius

\section{Einleitung}

Trotz der enormen Fortschritte der Frakturversorgung in den letzten Jahrzehnten heilen 5-10\% aller Knochenbrüche nur verzögert oder inkomplett aus [1]. Frakturheilungsstörungen beeinträchtigen die Lebensqualität und die Lebenserwartung der betroffenen Patienten und stellen ein erhebliches sozioökonomisches Problem dar.

Die Ursachen für die Entwicklung einer Frakturheilungsstörung sind vielfältig. Neben unzureichender Frakturstabilisierung, ungünstiger Reposition der Fragmente, mangelnder Durchblutung des Frakturgebiets oder Infektionen können weitere biologische Störgrößen, wie Begleitverletzungen und -erkrankungen, eine große Rolle spielen ( $\bullet$ Abb. 1).

\section{Pathophysiologie der gestörten Knochenbruchheilung}

Die Häufigkeit von Frakturheilungsstörungen wird je nach Literatur mit 5-10\% angegeben. Diese Zahlen schwanken sehr stark in Abhängigkeit der untersuchten Länder, des betroffenen Knochens und des Frakturtyps [2]. Zu den Frakturheilungsstörungen zählen eine verzögerte Frakturheilung und die Ausbildung einer Pseudarthrose. Benötigt ein Knochenbruch länger als 3 Monate bis zur Konsolidierung, spricht man von einer verzögerten Frakturheilung. Ist die Fraktur nach 6 Monaten noch immer nicht vollständig geheilt, liegt eine Pseudarthrose vor. Diese Zeiträume können je nach Frakturtyp variieren [3] und werden in verschiedenen Ländern unterschiedlich definiert.

\section{Ablauf der Frakturheilung}

Die physiologische Knochenheilung folgt einem komplexen Prozess mit kaskadenartig ablaufenden, ineinander übergehenden Phasen, die als Entzündungs-, Regenerations- und Remodeling-Phase bezeichnet werden. Entscheidend für eine erfolgreiche Knochenheilung sind, neben optimalen biomechanischen Gegebenheiten im Frakturgebiet und einer ausreichenden Durchblutung, streng regulierte und koordinierte Interaktionen zwischen den verschiedenen beteiligten Zelltypen, Zytokinen, Wachstumsfaktoren und weiteren biologischen Botenstoffen [1]. Die initiale Phase der Knochenheilung ist maßgeblich durch entzündliche Prozesse im Frakturgebiet gekennzeichnet. Durch den Knochenbruch kommt es zur Zerstörung von Gewebe, Blutgefäßen und Zellen. Dadurch bildet sich rund um die Bruchstelle ein Frakturhämatom, das durch einen niedrigen $\mathrm{pH}$-Wert, geringe Sauerstoffgehalte und eine hohe Konzentration an inflammatorischen Mediatoren gekennzeichnet ist. Durch diese lokalen Bedingungen werden Immunzellen zur Frakturstelle gelockt. Zunächst werden Zellen der angeborenen Immunantwort, wie neutrophile Granulozyten, Makrophagen und natürliche Killerzellen, rekrutiert. Später wandern auch Zellen der adaptiven Immunantwort, wie Tund B-Lymphozyten, in das Frakturhämatom ein [1]. In tierexperimentellen Studien konnte gezeigt werden, dass die Anwesenheit und physiologische Funktion von Immunzellen in der inflammatorischen Phase signifikant zum Heilungserfolg nach Fraktur beiträgt. So konnte in Tiermodellen eine gestörte bzw. veränderte Frakturheilung in Abwesenheit von neutrophilen Granulozyten [4], Makrophagen [5], Mastzellen [6] und Lymphozyten [7] nachgewiesen werden. Auch eine überschießende Entzündungsantwort wirkt sich negativ auf die Knochenheilung aus [1]. Immunzellen sind nicht nur für die inflammatorische Phase der Frakturheilung zuständig, sie interagieren auch mit wichtigen Zellen der regenerativen Phase. So modulieren Immunzellen unter anderem die Proliferation und Reifung von mesenchymalen Stromazellen (MSCs), die während der regenerativen Kallusentwicklung in Knorpel- und Knochenzellen differenzieren [8]. Die Knorpel- und Knochenbildung im Frakturkallus wird von einer Vielzahl von Zytokinen und Wachstumsfaktoren gesteuert [9]. Parallel zur Knochenformation entstehen neue Blutgefäße im Frakturkallus. Dieser Prozess der Neovaskularisierung wird maßgeblich durch angiogene Faktoren reguliert [10]. In der anschließenden Remodeling-Phase wird der externe Kallus durch Osteoklasten resorbiert und die ursprüngliche Knochenform und -architektur wiederhergestellt. Fehlen wichtige Botenstoffe oder ist die Funktion und Kommunikation der verschiedenen Zellarten im Frakturkallus gestört, kann es zu Heilungsstörungen kommen [11].

\section{Biologische Störfaktoren}

Häufig treten Frakturheilungskomplikationen im Patienten mit Begleitverletzungen oder -erkrankungen auf. In diesem Artikel soll auf die Störfaktoren schweres Trauma, 


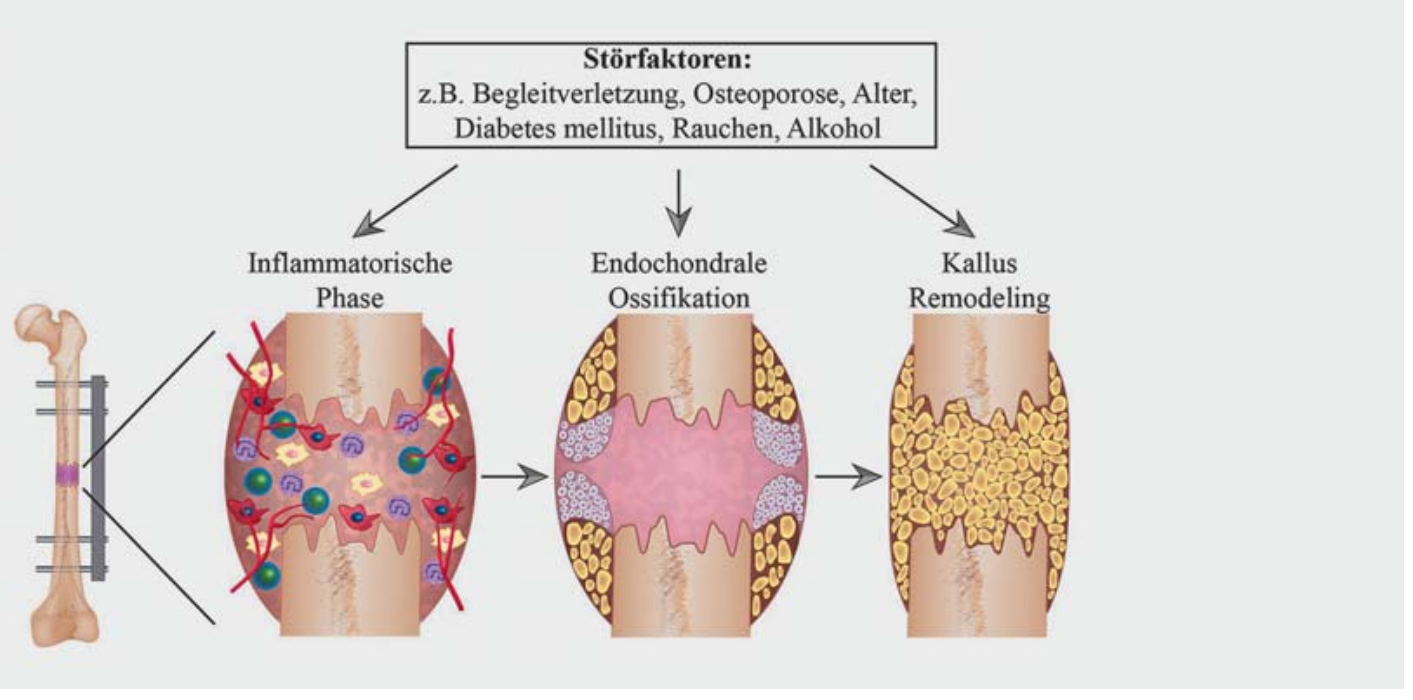

- Abb. 1 Darstellung der verschiedenen Phasen der Frakturheilung und möglicher Störfaktoren. Die Frakturheilung beginnt mit einer inflammatorischen Phase, die durch die Ruptur von Blutgefäßen und das Einwandern von inflammatorischen Zellen ins Frakturgebiet gekennzeichnet ist. Durch die Rekrutierung und Differenzierung von mesenchymalen Stromazellen wird der Prozess der endochondralen Ossifikation eingeleitet. Hierbei wird der initiale knorpelige Kallus zu Knochengewebe umgewandelt. Nach der knöchernen Überbrückung des Frakturspalts beginnt die Remodeling-Phase, in welcher der externe Frakturkallus abgebaut und die ursprüngliche Knochengeometrie und -struktur wiederhergestellt werden. Störfaktoren wie z. B. Begleitverletzungen, Osteoporose, Alter, Diabetes mellitus, Rauchen und übermäßiger Alkoholgenuss können alle Phasen der Knochenheilung beeinträchtigen.

Diabetes, Osteoporose, Alter, Rauchen und Alkoholabusus eingegangen werden. Diese Störfaktoren stellen aufgrund ihrer hohen Inzidenz in der Bevölkerung ein schwerwiegendes medizinisches Problem dar.

\section{Störfaktor Begleitverletzung}

Knochenbrüche, die mit Begleitverletzungen wie großen Weichteilschäden von Muskulatur und Periost einhergehen, zeigen oftmals signifikante Heilungsverzögerungen [12]. Schäden an den umliegenden Strukturen und versorgenden Blutgefäßen verursachen eine lokale Minderdurchblutung, die sich je nach Verletzungsschwere negativ auf die Frakturheilung auswirken kann. Fehlende Sauerstoffsättigung und Nährstoffversorgung durch mangelnde Durchblutung können eine physiologische Regeneration verhindern. Als ein weiterer Pathomechanismus der gestörten Heilung im Patienten mit großem Weichteiltrauma wird auch die verringerte Rekrutierung von Stammzellen aus den verletzten Geweben diskutiert $[13,14]$. Auch eine systemische Entzündungsantwort, ausgelöst durch schwere Verletzungen oder Mehrfachverletzungen, kann die Frakturheilung negativ beeinflussen. So konnte in Experimenten an Maus und Ratte gezeigt werden, dass ein zusätzliches Thoraxtrauma, das eine überschießende posttraumatische Immunantwort induziert, die Knochenheilung signifikant stört $[1,15]$. Die erhöhte systemische posttraumatische Inflammation sorgt für eine verstärkte Rekrutierung von Immunzellen ins Frakturhämatom, die sich negativ auf die Knochenregeneration auswirken kann. Wichtige Mediatoren, die in diesem Prozess beteiligt zu sein scheinen, sind Komplementanaphylatoxine und inflammatorische Zytokine. Das Komplementsystem gilt als posttraumatisches Frühwarnsystem und wesentlicher Auslöser einer systemischen Inflammationsantwort nach Trauma. Seine Funktion besteht unter anderem darin, exogene Pathogene und endogene Alarmsignale zu erkennen und darauf unter anderem mit der Bildung der Anaphylatoxine C3a und C5a zu reagieren. Diese Mediatoren besitzen ein ausgeprägtes proinflammatorisches Wirkspektrum. Beispielsweise erhöhen sie die Bildung und Sekretion eines weiteren Schlüsselmoleküls in der posttraumatischen Entzündungsreaktion, Interleukin-6. Dieses Molekül hat ein pleiotrophes Wirkspektrum und ist neben vielen weiteren Funktionen auch für die Rekrutierung von neutrophilen Granulozyten und T-Lymphozyten verantwortlich. Eine Dämpfung der Immunantwort nach Fraktur und Thoraxtrauma durch Inhibierung des Komplementrezeptors C5aR bzw. des Interleukin-6-Trans-Signalwegs zeigte positive Auswirkungen auf die Heilung des Knochens nach schwerem Trauma $[15,16]$. Auf der anderen Seite gibt es Hinweise darauf, dass eine andere Art der Begleitverletzung, das Schädel-Hirn-Trauma, die Knochenheilung beschleunigen kann [17]. Eine erhöhte Ausschüttung des neuroendokrinen Hormons Leptin, das osteoanabole Wirkung besitzt, wird als mögliche Ursache diskutiert [18]. Somit scheint die Art und die Schwere einer Begleitverletzung bei multiplem Trauma einen großen Einfluss auf die Knochenheilung zu haben. 


\section{Störfaktor Diabetes}

Diabetes mellitus ist eine Stoffwechselerkrankung, welche den Knochenmetabolismus stört und das Frakturrisiko erhöht [19]. Beide Arten des Diabetes mellitus (Typ 1 und Typ 2) beeinträchtigen die Frakturheilung über die Hyperglykämie, die erhöhte Bildung von „Advanced Glycation End Products" (AGE), die Bildung reaktiver Sauerstoffspezies (ROS) und eine erhöhte Entzündungsantwort [20]. Diese Faktoren führen zu einer erhöhten Osteoklasten- und einer verringerten Osteoblastenanzahl im intakten Knochen und im Frakturkallus. In vitro konnte gezeigt werden, dass hohe Glukosekonzentrationen ebenso wie AGEs die Aktivität von Osteoblasten über eine Inhibierung des osteoanabolen Wnt-Signalwegs reduzieren und die Bildung von Osteoklasten über eine erhöhte Expression des Zytokins Receptor Activator of NF-kB Ligand (RANKL) fördern [19]. Zusätzlich zu diesen molekularen Mechanismen ist im Patienten mit Diabetes häufig eine gestörte Durchblutung der Extremitäten zu finden. Dies wirkt sich ebenfalls negativ auf die Knochenheilung aus. Ein weiterer Mechanismus ist die erhöhte Expression von inflammatorischen Mediatoren in diabetischen Patienten. So konnte unter anderem gezeigt werden, dass in Mäusen mit Diabetes mellitus Typ 1 die Expression des Tumor Necrosis Factor $\alpha$ (TNF- $\alpha$ ) signifikant erhöht ist, was die endochondrale Frakturheilung negativ beeinflusst [21]. Eine Inhibierung von TNF- $\alpha$ verbesserte die Knochenheilung in diabetischen Mäusen und könnte eine mögliche therapeutische Intervention im diabetischen Patienten darstellen [21].

\section{Störfaktor Osteoporose}

Die häufigste Form der Osteoporose ist die postmenopausale Osteoporose, die bei $33 \%$ aller Frauen über 65 Jahren auftritt [22]. Sowohl im Patient als auch im Tiermodell ( $\mathbf{A} \mathbf{A b} \mathbf{b} . \mathbf{2}$ ) konnte bei Vorliegen einer postmenopausalen Osteoporose eine verzögerte Frakturheilung gezeigt werden $[23,24]$.

Allerdings wird in der Literatur kritisch diskutiert, ob osteoporotisches Knochengewebe per se schlechter heilt oder ob die im osteoporotischen Knochen schwer zu erzielende nötige Primärstabilität der Osteosynthese für die verzögerte Heilung sorgt. Grundsätzlich wirkt das Hormon Östrogen knochenanabol, was über seinen positiven Einfluss auf die Differenzierung von MSCs zu Osteoblasten und über seine negativen Effekte auf die Reifung und Aktivität von Osteoklasten vermittelt wird $[25,26]$. Analog dazu konnte in Mäusen während der Frakturheilung unter Östrogenmangel eine erhöhte Anzahl an Osteoklasten im Kallus festgestellt werden, was zu einer verringerten knöchernen Überbrückung des Frakturspalts führte [27]. Außerdem zeigte sich in östrogendefizienten Mäusen eine gestörte Entzündungsantwort nach Knochenbruch mit erhöhten Konzentrationen von proinflammatorischen Zytokinen, was ebenfalls zu einer gestörten Heilung beitragen könnte [28,29]. Supplementa-

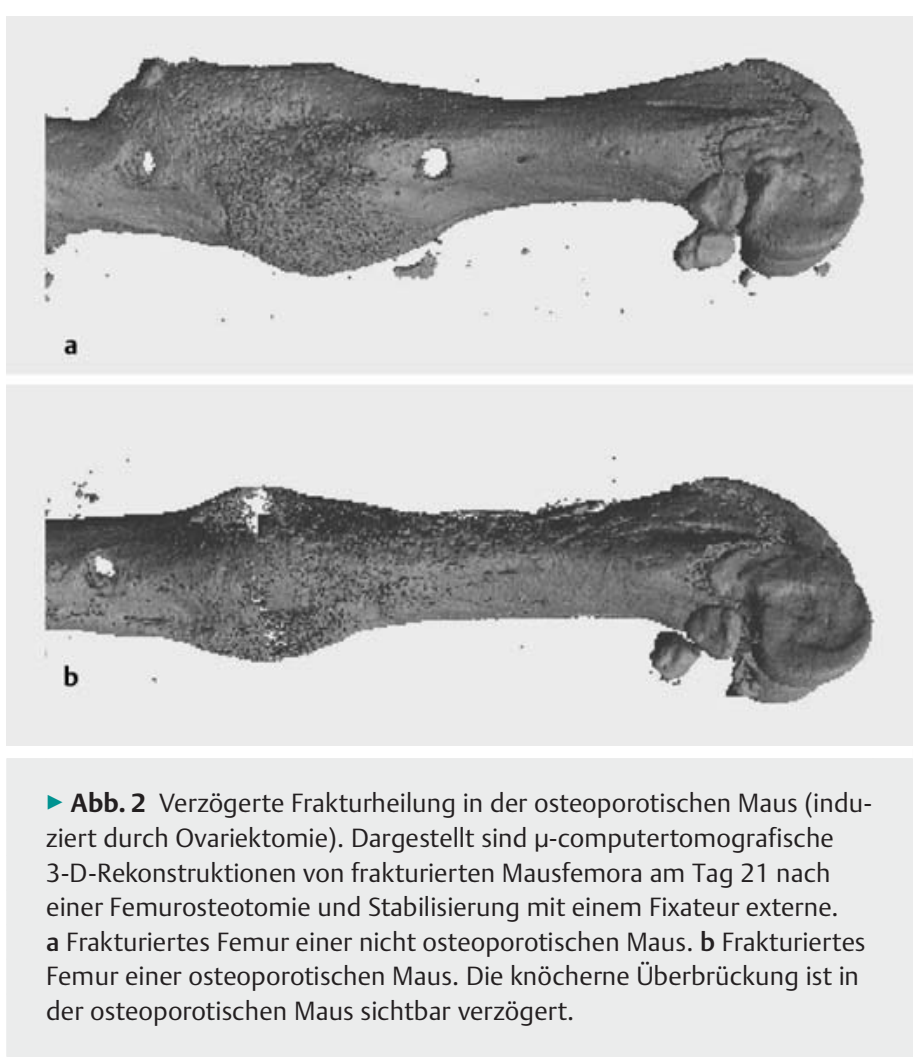

tion mit Östrogen, Östrogenanaloga oder selektiven Östrogen-Rezeptor-Modulatoren verbesserte die Frakturheilung im Tiermodell signifikant, was ebenfalls für einen negativen Effekt einer postmenopausalen Osteoporose auf die Frakturheilung spricht [24, 30].

\section{Störfaktor Alter}

In der Klinik zeigt sich eine Korrelation zwischen dem Alter des Patienten und einer gestörten Frakturheilung [31]. Frakturen heilen schneller in Kindern und jungen Erwachsenen als in älteren Patienten. Dies mag einerseits an der hohen Inzidenz für postmenopausale oder senile Osteoporose im alten Patienten liegen, andererseits nimmt die Regenerationsfähigkeit des Knochens im Alter ab [31]. Grundsätzlich umfasst Alterung den kontinuierlichen Verlust von vielen organspezifischen Funktionen und vermindert den Stammzellpool in den Organen, was die Regeneration des Gewebes nach Verletzungen verzögert. Die zugrunde liegenden Veränderungen finden auf unterschiedlichen zellulären und molekularen Ebenen statt: Die erhöhte Bildung von reaktiven Sauerstoffspezies und inflammatorischen Mediatoren im alten Organismus kann genetische und epigenetische Veränderungen auslösen, die wiederum die Proteinbiosynthese, Stammzellerneuerung und Zelldifferenzierung beeinträchtigen. Den Mitochondrien kommt eine zentrale Bedeutung in der Zell-, Organ- und Organismusalterung zu. Mitochondrien sind hauptsächlich für die endogene Bildung von reaktiven Sauerstoffspezies verantwortlich und steuern da- 
durch den Alterungsprozess maßgeblich [32]. Auch im Tiermodell konnte gezeigt werden, dass die Differenzierungskapazität von MSCs mit dem Alter sinkt und erhöhte Konzentrationen an proinflammatorischen Zytokinen und reaktiven Sauerstoffspezies die Knochenheilung stören können [31,33]. Zusammenfassend scheinen die molekularen und zellulären Mechanismen der gestörten Frakturheilung im alten Patienten sehr komplex zu sein. Mögliche therapeutische Interventionen umfassen die Stimulation der Kallusreifung mittels Wachstumsfaktoren wie Bone morphogenetic Protein 7 (BMP7), Tissue Growth Factor $\beta$ (TGF- $\beta$ ) und Insulin-like Growth Factor 1 (IGF-1), die sich in alten Nagern als effektiv zur Verbesserung der knöchernen Konsolidierung erwiesen haben [31].

\section{Störfaktor Rauchen}

Klinische Metaanalysen zeigen, dass Raucher ein 2-fach erhöhtes Risiko zur Ausbildung einer Pseudarthrose nach Fraktur haben [34]. Ebenso ist die Zeit bis zur vollständigen knöchernen Überbrückung bei Rauchern im Durchschnitt 1,5-mal so lang wie bei Nichtrauchern [34]. Kyro et al. führen dies auf eine Minderdurchblutung des verletzten Gewebes zurück und schlussfolgern daher, dass die negativen Auswirkungen von Rauchen auf die Knochenheilung in Patientengruppen mit per se gestörter Durchblutung, wie es z. B. in Diabetikern der Fall ist, verstärkt zum Tragen kommen [35]. Auch im Tiermodell konnte gezeigt werden, dass Rauchen die Knochenheilung verzögert. Mäuse, die täglich Zigarettenrauch ausgesetzt waren, zeigten eine signifikante Verzögerung der Knorpelentwicklung im Frakturkallus, was schließlich zu einer verschlechterten knöchernen Überbrückung führte [36]. Ein möglicher molekularer Mechanismus ist die verstärkte Bildung von reaktiven Sauerstoffspezies (ROS) und anderen Radikalen. In vitro konnte bereits gezeigt werden, dass Zigarettenrauch über die Bildung von ROS die osteogene und chondrogene Differenzierung von MSCs stört und die Zellproliferation hemmt [37]. Somit stellt Rauchen einen signifikanten Risikofaktor zur Ausbildung einer gestörten Knochenregeneration dar.

\section{Störfaktor Alkoholabusus}

Sowohl chronischer Alkoholismus als auch exzessives Trinken von Alkohol kurz vor einem Unfall führt zu einem erhöhten Risiko zur Ausbildung einer Pseudarthrose [38]. Dies hat eine hohe klinische Relevanz, da gerade bei jungen Patienten bis zu 40\% einen erhöhten Blutalkoholspiegel zum Zeitpunkt einer Fraktur aufweisen. Im Tiermodell konnte gezeigt werden, dass Alkoholadministration zu erhöhten Konzentrationen an ROS, inflammatorischen Zytokinen und einer verringerten Expression von osteoanabolen Wachstumsfaktoren führt und dadurch die Knochenheilung stört [39]. Besonders der osteoanabole Wnt-Signalweg scheint durch Alkoholabusus beeinträchtigt zu werden. So konnte im Tiermodell eine Aktivierung des Wnt-Signalwegs die negativen Effekte des Alkohols auf die Frakturheilung aufheben [39]. Eine weitere therapeutische Option bei alkoholbedingter Frakturheilungsverzögerung wäre die Applikation von endogenen Stammzellen. So zeigte die Injektion von MSCs im Tiermodell einen osteoanabolen Effekt und reduzierte die Effekte des Alkohols auf die Knochenheilung [40].

\section{Schlussfolgerungen}

Neben den klinischen Problemen einer unzureichenden Frakturstabilisierung, einer ungünstigen Reposition der Knochenenden, mangelnder Durchblutung oder des Auftretens von Infektionen können weitere biologische Störgrößen die Knochenbruchheilung signifikant beeinflussen. Insbesondere sind hier Begleitverletzungen, Komorbiditäten wie Diabetes und Osteoporose, fortgeschrittenes Alter und ungünstiger Lebensstil zu nennen. All diese Parameter sollten in Betracht gezogen werden bei der klinischen Einschätzung des Risikos einer verzögerten Heilung im individuellen Patienten. Auf Basis der molekularen Besonderheiten der jeweiligen Störfaktoren könnten in Zukunft individuelle therapeutische Strategien zur Verbesserung der Frakturheilung im Risikopatienten entwickelt werden.

\section{Interessenkonflikt}

Es besteht kein Interessenkonflikt.

Autorinnen/Autoren

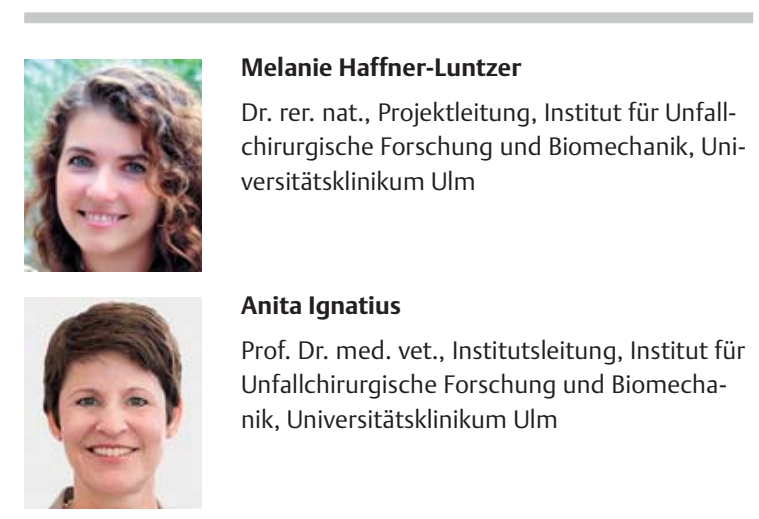

Korrespondenzadresse

Melanie Haffner-Luntzer
Institut für Unfallchirurgische Forschung und Biomechanik
Universitätsklinikum Ulm
Helmholtzstraße 14
89081 Ulm
Tel.: $0731 / 50055329$
Fax: $0731 / 50055302$
melanie.haffner-luntzer@uni-ulm.de


[1] Claes L, Recknagel S, Ignatius A. Fracture healing under healthy and inflammatory conditions. Nat Rev Rheumatol 2012; 8: 133-143. doi:10.1038/nrrheum.2012.1

[2] Bhandari M, Tornetta P 3rd, Sprague S et al. Predictors of reoperation following operative management of fractures of the tibial shaft. J Orthop Trauma 2003; 17: 353-361

[3] Marsh D. Concepts of fracture union, delayed union, and nonunion. Clin Orthop Relat Res 1998; 355: S22-S30

[4] Kovtun A, Bergdolt S, Wiegner $R$ et al. The crucial role of neutrophil granulocytes in bone fracture healing. Eur Cell Mater 2016; 32: 152-162

[5] Alexander KA, Chang MK, Maylin ER et al. Osteal macrophages promote in vivo intramembranous bone healing in a mouse tibial injury model. J Bone Miner Res 2011; 26: 1517-1532. doi:10.1002/jbmr.354

[6] Kroner J, Kovtun A, Kemmler J et al. Mast cells are critical regulators of bone fracture-induced inflammation and osteoclast formation and activity. J Bone Miner Res 2017; 32: 24312444. doi:10.1002/jbmr.3234

[7] Sun G, Wang Y, Ti Y et al. Regulatory B cell is critical in bone union process through suppressing proinflammatory cytokines and stimulating Foxp3 in Treg cells. Clin Exp Pharmacol Physiol 2017; 44: 455-462. doi:10.1111/1440-1681.12719

[8] Einhorn TA, Gerstenfeld LC. Fracture healing: mechanisms and interventions. Nat Rev Rheumatol 2015; 11: 45-54. doi:10.1038/nrrheum.2014.164

[9] Giannoudis PV, Einhorn TA, Marsh D. Fracture healing: the diamond concept. Injury 2007; 38 (Suppl. 4): S3-S6

[10] Lu C, Saless N, Wang X et al. The role of oxygen during fracture healing. Bone 2013; 52: 220-229. doi:10.1016/j.bone.2012. 09.037

[11] Abou-Khalil R, Colnot C. Cellular and molecular bases of skeletal regeneration: what can we learn from genetic mouse models? Bone 2014; 64: 211-221. doi:10.1016/j.bone.2014.03.046

[12] Gardner M], Higgins TA, Harvin WH et al. What Is Important Besides Getting the Bone to Heal? Impact on Tissue Injury Other Than the Fracture. J Orthop Trauma 2018; 32 (Suppl. 1): S21-S24. doi:10.1097/BOT.0000000000001125

[13] Shah K, Majeed Z, Jonason J et al. The role of muscle in bone repair: the cells, signals, and tissue responses to injury. Curr Osteoporos Rep 2013; 11: 130-135. doi:10.1007/s11914013-0146-3

[14] Henrotin Y. Muscle: a source of progenitor cells for bone fracture healing. BMC Med 2011; 9: 136. doi:10.1186/1741-70159-136

[15] Kaiser K, Prystaz K, Vikman A et al. Pharmacological inhibition of IL-6 trans-signaling improves compromised fracture healing after severe trauma. Naunyn Schmiedebergs Arch Pharmacol 2018; 391: 523-536. doi:10.1007/s00210-018-1483-7

[16] Recknagel S, Bindl R, Kurz J et al. C5 aR-antagonist significantly reduces the deleterious effect of a blunt chest trauma on fracture healing. J Orthop Res 2012; 30: 581-586. doi:10.1002/ jor.21561

[17] Brady RD, Grills BL, Church JE et al. Closed head experimental traumatic brain injury increases size and bone volume of callus in mice with concomitant tibial fracture. Sci Rep 2016; 6: 34491. doi:10.1038/srep34491

[18] Seemann R, Graef F, Garbe A et al. Leptin-deficiency eradicates the positive effect of traumatic brain injury on bone healing: histological analyses in a combined trauma mouse model. J Musculoskelet Neuronal Interact 2018; 18: 32-41
[19] Hamann C, Kirschner S, Gunther KP et al. Bone, sweet bone osteoporotic fractures in diabetes mellitus. Nat Rev Endocrinol 2012; 8: 297-305. doi:10.1038/nrendo.2011.233

[20] Jiao H, Xiao E, Graves DT. Diabetes and Its Effect on Bone and Fracture Healing. Curr Osteoporos Rep 2015; 13: 327-335. doi:10.1007/s11914-015-0286-8

[21] Kayal RA, Siqueira M, Alblowi J et al. TNF-alpha mediates diabetes-enhanced chondrocyte apoptosis during fracture healing and stimulates chondrocyte apoptosis through FOXO1. J Bone Miner Res 2010; 25: 1604-1615. doi:10.1002/jbmr.59

[22] Boonen S, Dejaeger E, Vanderschueren D et al. Osteoporosis and osteoporotic fracture occurrence and prevention in the elderly: a geriatric perspective. Best Pract Res Clin Endocrinol Metab 2008; 22: 765-785. doi:10.1016/j.beem.2008.07.002

[23] Nikolaou VS, Efstathopoulos N, Kontakis G et al. The influence of osteoporosis in femoral fracture healing time. Injury 2009; 40: 663-668. doi:10.1016/j.injury.2008.10.035

[24] Beil FT, Barvencik F, Gebauer M et al. Effects of estrogen on fracture healing in mice. J Trauma 2010; 69: 1259-1265. doi:10.1097/TA.0b013e3181c4544d

[25] Cheung WH, Miclau T, Chow SK et al. Fracture healing in osteoporotic bone. Injury 2016; 47 (Suppl. 2): S21-S26. doi:10.1016/S0020-1383(16)47004-X

[26] Shang ZZ, Li X, Sun HQ et al. Differentially expressed genes and signalling pathways are involved in mouse osteoblast-like MC3T3-E1 cells exposed to 17- $\beta$ estradiol. Int J Oral Sci 2014; 6: 142-149. doi:10.1038/ijos.2014.2

[27] Namkung-Matthai $H$, Appleyard R, Jansen J et al. Osteoporosis influences the early period of fracture healing in a rat osteoporotic model. Bone 2001; 28: 80-86

[28] Haffner-Luntzer M, Fischer V, Prystaz $\mathrm{K}$ et al. The inflammatory phase of fracture healing is influenced by oestrogen status in mice. Eur J Med Res 2017; 22: 23. doi:10.1186/s40001017-0264-y

[29] Fischer V, Kalbitz M, Muller-Graf F et al. Influence of Menopause on Inflammatory Cytokines during Murine and Human Bone Fracture Healing. Int J Mol Sci 2018; 19: E2070. doi:10.3390/ijms19072070

[30] Spiro AS, Khadem S, Jeschke A et al. The SERM raloxifene improves diaphyseal fracture healing in mice. J Bone Miner Metab 2013; 31: 629-636. doi:10.1007/s00774-013-0461-x

[31] Gruber R, Koch H, Doll BA et al. Fracture healing in the elderly patient. Exp Gerontol 2006; 41: 1080-1093. doi:10.1016/j.exger.2006.09.008

[32] Eming SA, Wlaschek M, Scharffetter-Kochanek K. [Wound healing in the elderly]. Hautarzt 2016; 67: 112-116. doi:10.1007/s00105-015-3738-2

[33] Clark D, Nakamura M, Miclau T et al. Effects of Aging on Fracture Healing. Curr Osteoporos Rep 2017; 15: 601-608. doi:10.1007/s11914-017-0413-9

[34] Pearson RG, Clement RG, Edwards KL et al. Do smokers have greater risk of delayed and non-union after fracture, osteotomy and arthrodesis? A systematic review with meta-analysis. BMJ Open 2016; 6: e010303. doi:10.1136/bmjopen-2015010303

[35] Kyro A, Usenius JP, Aarnio M et al. Are smokers a risk group for delayed healing of tibial shaft fractures? Ann Chir Gynaecol 1993; 82: 254-262

[36] El-Zawawy HB, Gill CS, Wright RW et al. Smoking delays chondrogenesis in a mouse model of closed tibial fracture healing. J Orthop Res 2006; 24: 2150-2158. doi:10.1002/jor.20263 
[37] Shaito A, Saliba J, Husari A et al. Electronic Cigarette Smoke Impairs Normal Mesenchymal Stem Cell Differentiation. Sci Rep 2017; 7: 14281. doi:10.1038/s41598-017-14634-z

[38] Duckworth AD, Bennet SJ, Aderinto J et al. Fixation of intracapsular fractures of the femoral neck in young patients: risk factors for failure. J Bone Joint Surg Br 2011; 93: 811-816. doi:10.1302/0301-620X.93B6.26432

[39] Roper PM, Abbasnia P, Vuchkovska A et al. Alcohol-related deficient fracture healing is associated with activation of FoxO transcription factors in mice. J Orthop Res 2016; 34: 21062115. doi:10.1002/jor.23235
[40] Obermeyer TS, Yonick D, Lauing K et al. Mesenchymal stem cells facilitate fracture repair in an alcohol-induced impaired healing model. J Orthop Trauma 2012; 26: 712-718. doi:10.1097/BOT.0b013e3182724298

Bibliografie

DOI https://doi.org/10.1055/a-0677-3615

Online-publiziert 30.11.2018 | OP-JOURNAL 2019; 35: 5-10 (c) Georg Thieme Verlag KG Stuttgart · New York ISSN 0178-1715 